\title{
在有机化学实验中设置计算化学模块
}

李田, 张攀, 边砧, 关玲, 徐烜峰, 张奇涵 ${ }^{*}$, 王婕妤 ${ }^{*}$, 王颖霞

北京大学化学与分子工程学院, 化学基础实验教学中心, 化学教育研究所, 北京 100871

摘要: 计算化学快速发展, 对于化学研究有非常强的指导作用, 因此将其引入本科低年级实验教学很有必要。本文 在调研国内外高校计算化学课程开设情况的基础上, 根据本院学生特点和课程的需要, 在面向大一本科生的核心实 验课程一有机化学实验中引入了计算模块。模块设计从实验问题出发, 将计算项目和实验紧密结合, 采用分层次 教学的模式进行。利用 Canvas 线上教学平台对微课、讲义、作业、测验等学习资源进行有效组织, 通过直播课和 微信群进行讨论和答疑, 以翻转课堂的形式进行混合式教学, 取得了很好的教学效果。

关键词：计算化学；有机化学实验; Gaussian 03 程序; 翻转课堂; 微课; Canvas 教学平台 中图分类号: G64; O6

\section{Computational Exercises in Undergraduate Organic Chemistry Laboratory}

Tian Li, Pan Zhang, Lei Bian, Ling Guan, Xuanfeng Xu, Qihan Zhang *, Jieyu Wang *, Yingxia Wang * Institute for Chemical Education, Experimental Chemistry Center, College of Chemistry and Molecular Engineering, Peking University, Beijing 100871, China.

\begin{abstract}
The development of computer technology has promoted the application of computational chemistry teaching in undergraduate Laboratory. We have introduced computation module into our "organic chemistry laboratory", which, as one of the core courses, is set up for fresh undergraduate students. The calculation is carried out by Gaussian and Gauss View, and the results are used in understanding the experimental phenomena. Canvas teaching platform was applied to organize the materials, including videos, handouts, quiz, homework and discussion, and a flipping teaching type was adopted to improve the study efficiency. A course survey showed that the combination of the computation with lab work are very welcome by the students and obtained a satisfactory result.
\end{abstract}

Key Words: Computational chemistry; Organic chemistry laboratory; Gaussian 03 program;

Flipped classroom; Micro-course; Canvas platform

近年来, 计算化学的研究伴随着计算机软硬件技术的进步而有了飞速发展, 并逐步成为与理论化 学、实验化学并重的化学研究支柱。化学发展到今天, “Chem” 已不再是单纯地根据经验去 “try”, 而是依据化学基本理论、运用计算化学这一越来越强大的工具, 对反应、结构和性质进行定量半定量 的解释和预测, 计算化学对实验的指导性已越来越强, 化学已经进入了一个崝新的阶段。当使用 “web of science” 来检索 “计算化学” 这个词时, 我们发现该领域的研究论文数量增长迅速, 这也正是计算 化学快速发展的佐证。目前, 我院本科教学中, 该部分内容主要在理论课程中讲授, 而计算化学作为 一种工具, 更多的需要在实验实践中运用, 因此, 在实验教学中引入计算化学模块十分必要。

收稿: 2020-12-22; 录用: 2020-12-29; 网络发表: 2020-12-29

“通讯作者, Emails: zqh@pku.edu.cn (张奇涵); jieyuwang@pku.edu.cn (王婕妤); yxwang@pku.edu.cn (王颖霞)

基金资助: 北京大学 2020 年教学新思路 2.0 项目; 北京大学 2020 年设备部实验教学改革项目 


\section{1 国内外高校计算化学实验的开设情况调研}

为了更好地进行教学设计, 我们首先对国内外高校中计算化学实验的开设情况进行了调研。国 内调研主要来源于中国知网的检索和与兄弟高校的交流, 国外调研主要来源于美国化学会知名教学 期刊Journal of Chemical Education 的检索, 以及国外高校官网的查询。调研结果显示, 在目前高校的 本科教学中, 计算实验的开设主要分为以下三种类型。

\section{1 独立设课, 开设专门的计算化学实验}

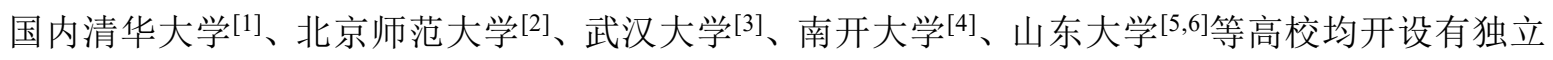
的计算化学实验课程。独立设课的优点在于, 可以完整地介绍计算化学的内容, 系统地进行实验训 练, 帮助学生获得扎实的基础。由于具有一定的系统性, 课程通常需要在高年级来开设。

\section{2 融入高年级的理论课程或实验课程, 作为其中的项目进行开设}

在高年级理论或实验课程中融入计算化学实验, 是另一种主要的开设方式。以加拿大滑铁卢大 学为例, 该校将苯甲酸和苯酚衍生物气相电离的Gibbs自由能变 $\Delta G$ 计算引入高年级的物理有机实验 中, 帮助学生理解取代基与反应活性之间的相互作用 ${ }^{[7]}$ 。西班牙拉古纳大学将电化学方法实现材料 表面接枝功能化引入高年级的物理化学实验, 计算了 HOMO和LUMO轨道的能量, 并绘制了分子的 电荷密度分布图, 帮助学生深入理解表面接枝功能化的机理和过程 ${ }^{[8]}$; 国外其他将计算融入高年级 理论课和实验课的实例还有很多 ${ }^{[9-11]}$, 我们中心也在 2015 年将Gaussian程序计算燃烧热和AMBER程 序模拟蛋白质结构的动态变化两个项目引入了面向大三本科生的物理化学实验中, 取得了很好的教 学效果。

\section{3 融入低年级的理论课程或实验课程, 作为其中的项目进行开设}

与高年级课程中引入计算实验有所不同, 在低年级课程, 尤其是实验课程中引入计算的范例仍 然很少。Journal of Chemical Education期刊中仅查到美国树城州立大学和威斯康辛大学麦迪逊分校 开展相关的教学工作 ${ }^{[12-15]}$ 。虽然目前这种模式较 “小众” ，但提供了一种新的开设思路。

综合国内外高校计算实验的开设情况, 结合我院学生基础好、学习能力强的特点, 我们认为在 低年级, 尤其是大一年级实验课程中开设计算模块值得尝试。引入计算内容, 不仅可以为学生提供 更加多元化的学习体验, 而且有助于学生及早对计算建立概念, 有意识地在后续学习中将计算化学 作为工具来解决实际问题, 同时有助于学生对抽象概念建立具象认识, 在后续学习结构化学、量子 化学等较为深奥的理论课程时, 化繁为简, 达到事半功倍的学习效果。

\section{2 计算化学实验的内容设计}

通过借鉴国内外兄弟院校的优秀经验, 并结合目前有机化学实验中开设的具体实验项目, 我们 设计了以下三项计算内容: (1) 通过三组分实验中苯胺分子的性质计算, 掌握量子化学计算的一般 流程; (2) 通过苯基肉桂酸合成实验中两个产物分子的计算, 学习如何运用计算工具合理解释实验 结果; (3) 通过傅-克反应不同进攻基团的计算, 掌握如何运用计算来理解反应和过渡态, 从而完成 计算能力的进阶。在计算项目的设计上, 我们力求将计算和实验相结合, 使计算真正为解决实验问 题服务, 帮助学生做到学以致用。详细的项目设计请见图1。

学生通过该模块的学习, 将获得以下几方面的能力:

(1) 具备初步的计算化学理论背景, 熟悉Gaussian和GaussView 软件的操作流程, 能够正确创建 初始结构、编写输入文件、执行计算过程、输出计算数据、规范表达计算结果;

(2) 学会如何针对实验问题设计计算方案;

(3) 学会如何运用计算结果合理解释实验问题。

由于Gaussian程序是目前使用最为广泛的量子化学计算软件之一, 因此, 课程中我们选用 Gaussian软件来开展计算化学实验的教学。 


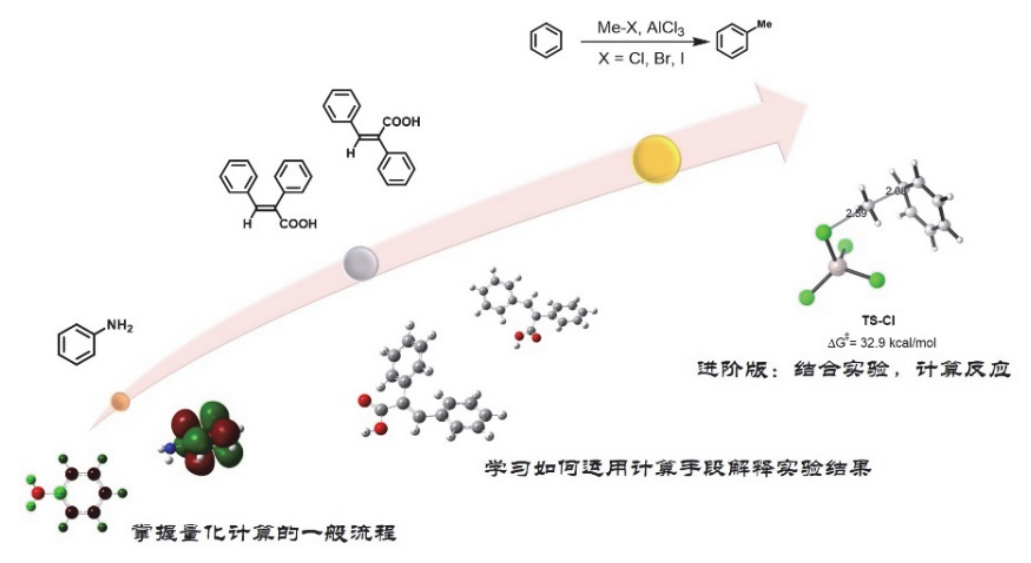

图1 计算实验模块的项目设计

\section{3 计算化学实验的教学模式}

\section{1 分层次教学, 满足学生的不同需求}

考虑到学生的基础和兴趣有所差别, 我们将计算项目分为必做和选做两部分, 为学生提供充分 的自由度。提高班学生要完成 3 个计算项目的学习, 常规班学生可自由选择学习其中 $1-2$ 个, 或仅仅 出于兴趣前来旁听(图2)。为学生提供自主选择的机会, 可以充分调动学生们的学习热情, 在学习过 程中遇到困难也会全力以赴, 取得更好的学习效果。

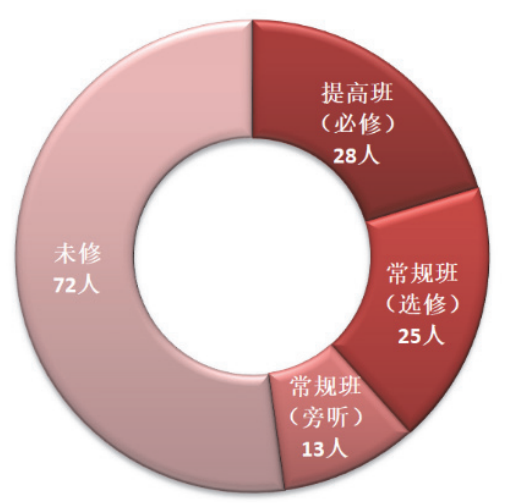

图2 选择计算实验模块的学生人数分布

\section{2 基于Canvas平台, 以翻转课堂的形式实现混合式教学}

Canvas学习平台因其强大的资源管理功能和完善的交互功能为国外多所藤校所采用。该项目中, 我们也采用Canvas平台来完成计算实验项目的建课。同时配合腾讯会议重难点串讲和微信群讨论答 疑来完善教学过程, 最后通过追踪学生的实验报告、在线测验结果和问卷反馈来综合评估学生的学 习效果。整体的教学设计如图3所示。

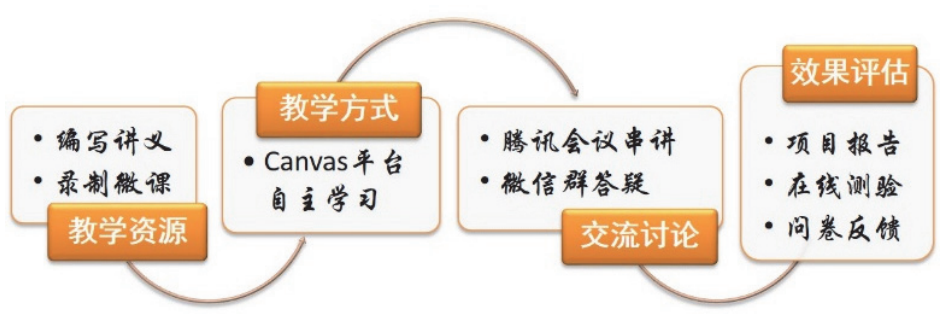

图3 教学流程设计 
讲义部分共计 7 个章节, 从计算化学是什么、能算什么、怎么算进行了详细的介绍。讲义中所有 和软件操作相关的部分均采用图文结合的方式, 力求内容清晰明了; 微课部分总计 $120 \mathrm{~min}$, 对分子 模型的构建、程序的编写和程序的运行进行了重点演示, 和讲义相互配合, 为学生提供多样化的学 习资源(图4); 作业部分回归到项目内容本身, 运用讲义和微课中学到的知识对具体的实验问题进行 计算, 从计算的角度做出合理解释; 项目测验环节则以考促学, 帮助学生们查漏补缺, 更好的掌握 学习内容。
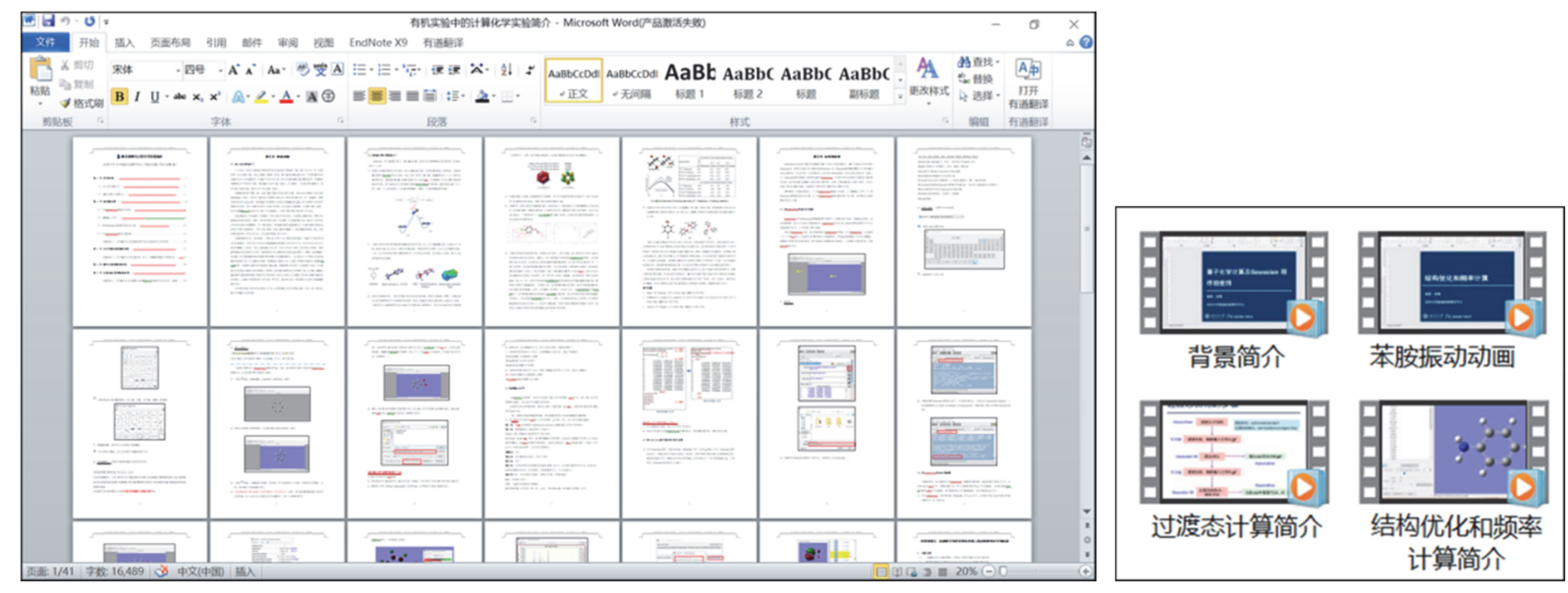

图4 建设完成的讲义和微课学习资源

以上学习资源经由Canvas教学平台进行有效组织。依照教学项目和学习进度, 我们将学习资源 分为三个部分, 每部分均包含阅读材料、学习视频和作业环节, 每周完成一个部分的学习, 最后一 周还会安排专门的测验。所有作业均提交电子版, 助教直接在Canvas平台上进行批改, 及时便捷, 且能保证学生获得实时反馈。建课后的Canvas平台如图5所示。

为了给学生提供更加精细化的指导, 每周我们特别安排助教和学生召开 “面对面” 的线上会议。 助教通过对讲义和微课的重难点进行集中讲解, 帮助学生梳理知识点, 强化内容理解。此外, 助教 还会逐一演示程序编写和软件使用。

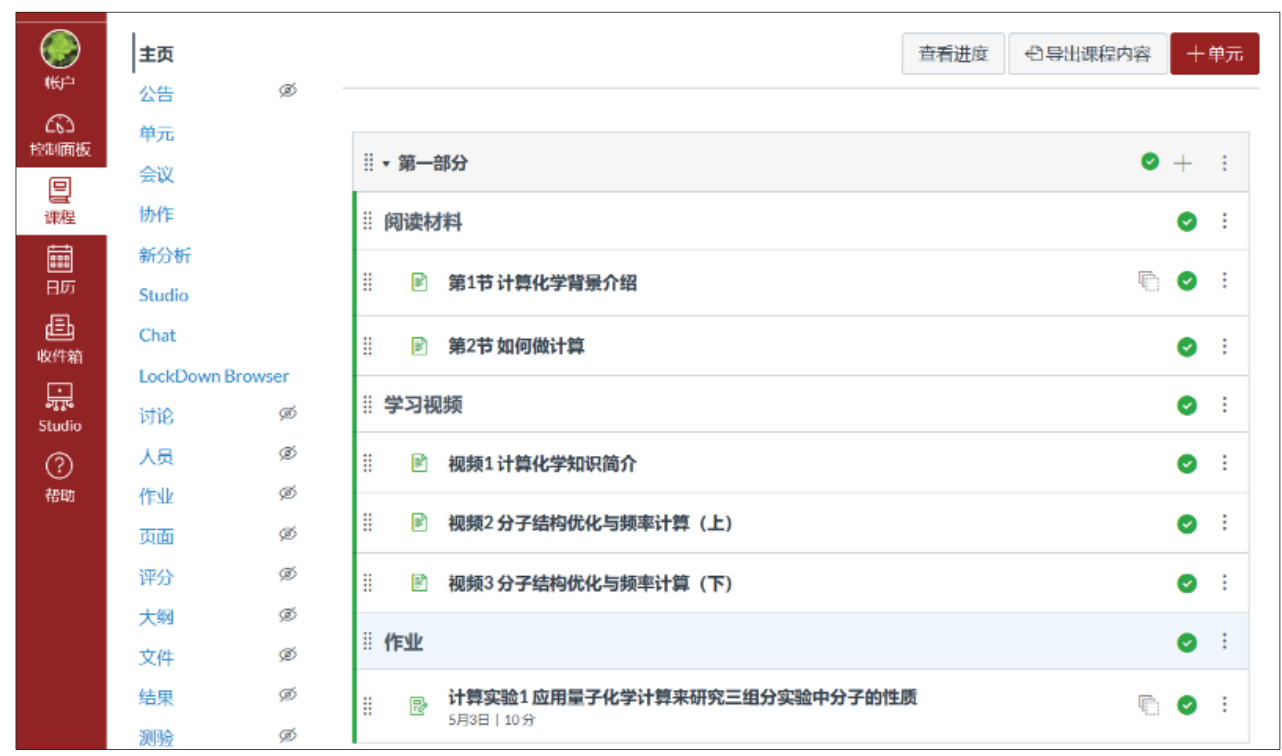

图5 完成建课的Canvas教学平台 
受疫情影响, 春季学期学生都远程在家学习, 为了实现实时零距离的沟通, 我们特别为主讲教 师、助教和学生建立了微信群。学生在学习过程中遇到任何问题都可随时提问, 老师和助教均第一 时间回复。除老师和助教全情投入外, 我们也鼓励学生之间相互交流和答疑, 在讨论中碰撞火花, 共同进步。

“Canvas平台线上自学+直播课重难点梳理+微信群讨论答疑” 以翻转课堂的形式在疫情期间顺 利完成了教学开展, 这也是我们基于混合式教学做出的一次成功尝试。

此外, 为了提高计算效率, 使学生获得更好的学习体验, 帮助学生对学校的计算资源有更加全 面的了解, 我们申请开通了学校计算中心超算平台的权限, 学生可以远程将计算任务提交到平台进 行运算, 及早接触和熟悉前沿的平台运算环境。

\section{4 教学效果评价和反馈}

为了进一步了解学生的在线学习效果, 获得更多的反馈建议, 我们特别设计问卷进行了调研。 提高班收回问卷反馈 25 份, 回收率达 $89 \%$, 常规班收回问卷反馈 22 份, 回收率达 $88 \%$ 。问卷共设置了 6 个问题, 内容涵盖计算项目难易程度、学习资源丰富程度、助教串讲和答疑的效果、对前沿类拓展 资源的需求、对应用计算工具解决实际问题的认识等。给予我们鼓励的是, 提高班和常规班学生在 所有问题上均给出了超过 $95 \%$ 的正面反馈, 尤其在问题 “我想把计算实验推荐给学弟学妹, 鼓励他 们来修习” 中, 全体同学更是 $100 \%$ 给出了同意的观点(图6)。从问卷结果来看, 项目取得了很好的教 学效果, 达到了教学预期。

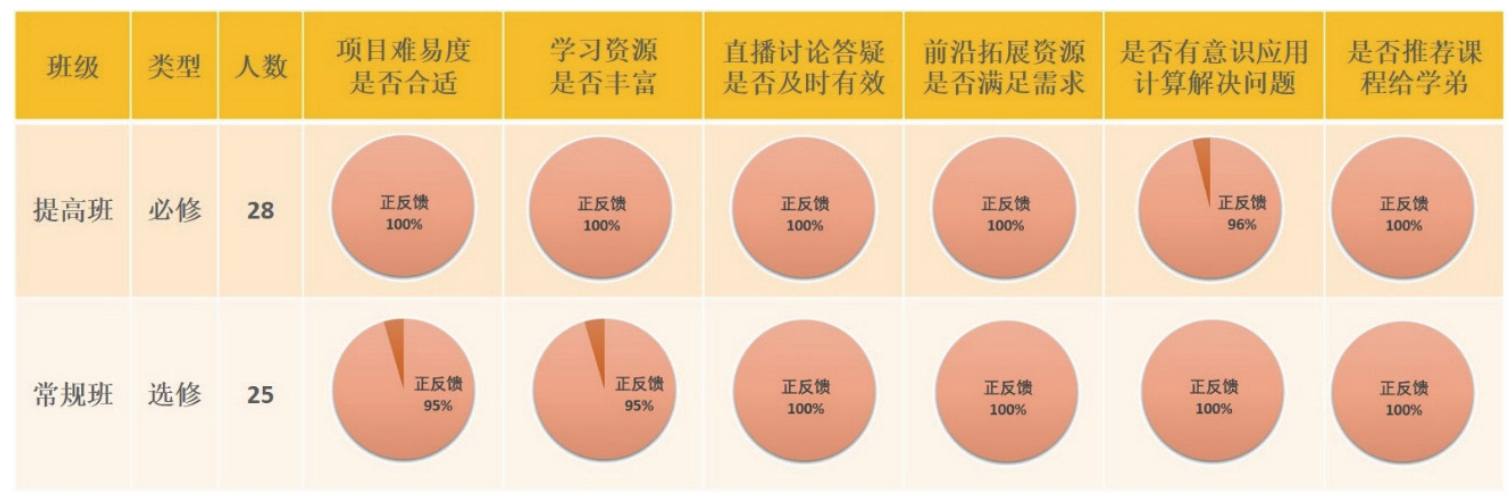

图6 计算化学模块问卷反馈

\section{5 教学总结与后续计划}

该项目于2020年春季学期首次开设, 从学生反馈来看, 计算化学实验模块的内容建设较为完善, 实现了既定的教学目标, 并获得了学生们的普遍认可。经过本轮教学后, 我们对学生的学习情况和 需求也有了更深入的了解, 后续我们将从以下几方面对课程做进一步完善：1) 在资源拓展和科研衔 接方面做进一步努力: 将整理计算化学领域的前沿文献作为拓展资料分享给学生阅读; 2) 推荐精编 教材, 汇编优质学习资源, 补充推荐给学生做参考; 3) 继续丰富和完善计算项目, 引入动力学计算、 $\mathrm{NBO}$ 分析等计算内容; 4) 后续开课时, 将推广开来, 面向有机实验的所有学生开设基本的计算内容, 同时继续以分层次教学的模式为学生提供多样化学习方案, 力求将计算化学学习更加有效地贯穿于 学生的实验学习中。

致谢：感谢余子迪和丁丽两位研究生助教对本工作的支持。 
[1] 王溢否, 李隽. 大学化学, 2018, $33(10), 25$.

[2] 胡红智, 马思渝. 计算化学实验. 北京: 北京师范大学出版社, 2008 .

[3] 王宝山, 侯华. 分子模拟实验. 北京: 高等教育出版社, 2010.

[4] 许秀芳. 大学化学, 2017, 32 (2), 23.

[5] 张恒, 马芗, 刘刚, 张冬菊, 宋其圣, 苑世领. 大学化学, 2019, 34 (9), 26.

[6] 苑世领, 张恒, 张冬菊. 分子模拟一理论与实验. 北京: 化学工业出版社, 2016.

[7] Blake, E. Z. J. Chem. Educ. 2013, 90, 665.

[8] Miriam, C. R. G.; Alberto, H. C.; Pilar. C. J. Chem. Educ. 2018, 95, 1386.

[9] Anna, M. F.; Megan, J. T. J. Chem. Educ. 2014, 91, 2191.

[10] Carrie, S. M.; Mark, E. J. Chem. Educ. 2015, 92, 1040.

[11] Rodríguez Ortega, P. G.; Montejo, M.; Valera, M. S.; López González, J. J. J. Chem. Educ. 2019, 96, 1760.

[12] Clifford, M. C.; Jeremy, P. D.; Lauren, E. D.; Tyler, P. H.; Karen, A. H.; Owen, M. M.; Don, L. W. J. Chem. Educ. 2013, 90, 1235.

[13] Allen, D. C.; Stephen, F. N. J. Chem. Educ. 2009, 86, 955.

[14] Brian, J. E.; Nicholas, J. H. J. Chem. Educ. 2016, 93, 932.

[15] Maria, A. Z.; Cara, E. S.; Asif, D. H.; Nicholas, J. H.; Brian, J. E. J. Chem. Educ. 2018, 95, 1960. 